\title{
Diagnostic performance and prediction of clinical progression of plasma phospho-tau181 in the Alzheimer's Disease Neuroimaging Initiative
}

Running title: Diagnostic and prognostic performance of plasma p-tau181

Thomas K. Karikari ${ }^{1 \dagger}$, Andréa L. Benedet ${ }^{2 \dagger}$, Nicholas J. Ashton ${ }^{1,3,4,5 \dagger}$, Juan Lantero Rodriguez ${ }^{1}$, Anniina Snellman ${ }^{1,6}$, Marc Suárez-Calvet ${ }^{7,8,9,10}$, Paramita Saha-Chaudhuri ${ }^{11}$, Firoza Lussier ${ }^{2}$, Hlin Kvartsberg ${ }^{1,12}$, Alexis Moscoso Rial ${ }^{1,3}$, Tharick A. Pascoal ${ }^{2,13}$, Ulf Andreasson ${ }^{1,12}$, Michael Schöll ${ }^{1,3,14}$, Michael W. Weiner ${ }^{15}$, Pedro RosaNeto $^{2,13}$, John Q. Trojanowski ${ }^{16,17 *}$, Leslie M. Shaw ${ }^{17}$, Kaj Blennow ${ }^{1,12 \ddagger}$, Henrik Zetterberg ${ }^{1,12,14,18 \sharp^{*}}$ for the Alzheimer's Disease Neuroimaging Initiative**

${ }^{1}$ Department of Psychiatry and Neurochemistry, Institute of Neuroscience and Physiology, The Sahlgrenska Academy, University of Gothenburg, Sweden; ${ }^{2}$ Translational Neuroimaging Laboratory, The McGill University Research Centre for Studies in Aging, H4H 1R3, Montreal, Canada; ${ }^{3}$ Wallenberg Centre for Molecular and Translational Medicine, University of Gothenburg, Sweden; ${ }^{4}$ King's College London, Institute of Psychiatry, Psychology \& Neuroscience, Maurice Wohl Clinical Neuroscience Institute, London, UK; ${ }^{5}$ NIHR Biomedical Research Centre for Mental Health \& Biomedical Research Unit for Dementia at South London \& Maudsley NHS Foundation, London, UK; ${ }^{6}$ Turku PET Centre, University of Turku, Kiinamyllynkatu 4-8, FI-20520 Turku, Finland; ${ }^{7}$ Barcelonaßeta Brain Research Center (BBRC), Pasqual Maragall Foundation. Barcelona, Spain; ${ }^{8}$ IMIM (Hospital del Mar Medical Research Institute), Barcelona, Spain; ${ }^{9}$ Servei de Neurologia, Hospital del Mar, Barcelona, Spain; ${ }^{10}$ Centro de Investigación Biomédica en Red de Fragilidad y Envejecimiento Saludable (CIBERFES), Madrid, Spain; ${ }^{11}$ Department of Epidemiology and Biostatistics, McGill University, Montreal, Québec, Canada; ${ }^{12} \mathrm{Clinical}$ Neurochemistry Laboratory, Sahlgrenska University Hospital, Mölndal, Sweden; ${ }^{13}$ Montreal Neurological Institute, H3A 2B4, Montreal, Canada; ${ }^{14}$ Department of Neurodegenerative Disease, UCL Institute of Neurology, London,

UK; ${ }^{15}$ Department of Radiology, Medicine, and Psychiatry, University of California San Francisco, San Francisco, CA, USA; ${ }^{16}$ Department of Pathology and Laboratory Medicine, Perelman School of Medicine, University of Pennsylvania, Philadelphia, PA, USA; ${ }^{17}$ Institute on Aging, Center for Neurodegenerative Disease Research, University of Pennsylvania School of Medicine, Philadelphia, PA, USA; ${ }^{18}$ UK Dementia Research Institute at UCL, London, UK.

${ }^{\dagger}$ Equal contribution as first authors

${ }^{\ddagger}$ Equal contribution as senior authors

**Data used in preparation of this article were obtained from the Alzheimer's Disease Neuroimaging Initiative

(ADNI) database (adni.loni.usc.edu). As such, the investigators within the ADNI contributed to the design and implementation of ADNI and/or provided data but did not participate in analysis or writing of this report. A complete listing of ADNI investigators can be found at: http://adni.loni.usc.edu/wpcontent/uploads/how to_apply/ADNI_Acknowledgement_List.pdf 
*Corresponding author: Professor Henrik Zetterberg, Department of Psychiatry and Neurochemistry, Institute of Neuroscience and Physiology, The Sahlgrenska Academy, University of Gothenburg, Mölndal, Sweden

Tel: +46 31-343 0142

Email: henrik.zetterberg@clinchem.gu.se

\section{Abstract}

Whilst cerebrospinal fluid (CSF) and positron emission tomography (PET) biomarkers for amyloid- $\beta$ (A $\beta$ ) and tau pathologies are accurate for the diagnosis of Alzheimer's disease (AD), their broad implementation in clinical and trial settings are restricted by high cost and limited accessibility. Plasma phosphorylated-tau181 (p-tau181) is a promising blood-based biomarker that is specific for $\mathrm{AD}$, correlates with cerebral $\mathrm{A} \beta$ and tau pathology, and predicts future cognitive decline. In this study, we report the performance of p-tau181 in >1,000 individuals from the Alzheimer's Disease Neuroimaging Initiative (ADNI), including cognitively unimpaired (CU), mild cognitive impairment (MCI) and $\mathrm{AD}$ dementia patients characterized by $\mathrm{A} \beta$ PET. We confirmed that plasma p-tau181 is increased at the preclinical stage of Alzheimer and further increases in $\mathrm{MCI}$ and $\mathrm{AD}$ dementia. Individuals clinically classified as AD dementia but having negative $\mathrm{A} \beta$ PET scans show little increase but plasma $p$-tau181 is increased if CSF A $\beta$ has already changed prior to A $\beta$ PET changes. Despite being a multicenter study, plasma p-tau181 demonstrated high diagnostic accuracy to identify $\mathrm{AD}$ dementia ( $\mathrm{AUC}=85.3 \%$; $95 \% \mathrm{CI}, 81.4 \%-89.2 \%$ ), as well as to distinguish between $\mathrm{A} \beta$ - and $\mathrm{A} \beta+$ individuals along the Alzheimer's continuum ( $\mathrm{AUC}=76.9 \%$; 95\% $\mathrm{CI}, 74.0 \%$ $79.8 \%$ ). Higher baseline concentrations of plasma p-tau181 accurately predicted future dementia and performed comparably to the baseline prediction of CSF p-tau181. Longitudinal measurements of plasma p-tau181 revealed low intra-individual variability, which could be of potential benefit in disease-modifying trials seeking a measurable response to a therapeutic target. This study adds significant weight to the growing body of evidence in the use of plasma p-tau181 as a non-invasive diagnostic and prognostic tool for $\mathrm{AD}$, regardless of clinical stage, which would be of great benefit in clinical practice and a large cost-saving in clinical trial recruitment. 


\section{Introduction}

Alzheimer's disease (AD) is the most common form of neurodegenerative dementia and is defined by the accumulation of amyloid beta $(\mathrm{A} \beta)$ and tau aggregates in the brain [1]. These pathological changes occur several years before the manifestation of clinical symptoms [2] and are initiated by the build of extracellular amyloid beta (A $\beta$ ) plaques, followed by the accumulation of aggregated phosphorylated tau (p-tau). AD dementia is most typically diagnosed based on clinical criteria; however, $A \beta$ pathology can now be accurately measured using $A \beta$ positron emission tomography (PET) or cerebrospinal fluid (CSF) A $\beta 42 / A \beta 40$ ratio [3]. Tau pathology can also be detected by tau PET or CSF concentration of tau phosphorylated at threonine-181 (p-tau181), which are both highly specific for AD [4]. Despite being accurate for AD diagnosis and prognosis [5-7], molecular neuroimaging and CSF biomarkers for $\mathrm{AD}$ have significant drawbacks for routine use in clinical settings including being invasive, timeconsuming and expensive. Furthermore these biomarkers are difficult to access, particularly in a primary care setting or remote communities [8]. Blood biomarkers may hold promise to address the challenges of these current methods.

Methods for the detection of $A \beta$ peptides in blood are available, the results of which are related to cerebral $A \beta$ pathology [9-11]. However, extracerebral expression of $A \beta$ peptides challenges their use in clinical laboratory practice. Blood biomarkers for tau have been lacking. While ultrasensitive plasma t-tau assays can detect neuronal injury in acute brain disorders, e.g., stroke and traumatic brain injury, they work relatively poorly in AD settings, and the correlation with CSF t-tau is weak [12]. Assays for the quantification of p-tau181 in blood have been recently developed and the results showed increased concentrations in AD dementia [13-16]. In cross-sectional single-center studies, blood p-tau181 was increased across the Alzheimer's clinical continuum - from preclinical Alzheimer to $\mathrm{AD}$ dementia - and enabled the differential diagnosis of $\mathrm{AD}$ as compared to non- $\mathrm{AD}$ neurodegenerative disorders [13, 15, 17-20]. Furthermore, plasma p-tau181 correlates strongly with CSF p-tau181 and PET measures of $A \beta$ and tau pathologies $[13,15,17,18]$. However, routine clinical applications of plasma ptau181 biomarkers would require a demonstration of robust validation in large multicenter studies. Furthermore, whilst our group showed that baseline plasma p-tau181 associates with cognitive decline and neurodegeneration one year later [13], it is unknown if baseline and serial measures of plasma p-tau181 can predict future progression to dementia in a larger cohort of individuals followed over longer periods. The relevance of plasma p-tau181 for monitoring the clinical and pathological progression of $\mathrm{AD}$ is unclear owing to the lack of longitudinal data. Addressing these knowledge gaps is critical for determining the suitability of using plasma p-tau181 for population screening, diagnosis, and as a recruitment and outcome measure for clinical trials [21].

In this study, we have investigated plasma p-tau181 in the Alzheimer's Disease Neuroimaging Initiative (ADNI) cohort. We examined: (i) how plasma p-tau181 performs diagnostically in a large multicenter study to verify findings from recent single-center studies of plasma p-tau181 [13,17, 18], (ii) how the biomarker performs in a head-to-head comparison with CSF biomarkers (p-tau181 and $\mathrm{A} \beta_{42}$ ), MRI and plasma NfL, (iii) if baseline plasma p-tau181 concentration is predictive of cognitive decline and conversion to $\mathrm{AD}$ dementia, and (iv) how longitudinal trajectories of plasma p-tau181 reflect stages of the AD continuum. 


\section{Materials and methods}

\section{Study participants}

We used data from the multicenter ADNI study designed to develop and validate neuroimaging and biochemical biomarkers for the early detection, monitoring and treatment of AD [22]. This North American cohort recruited participants across 57 sites in the US and Canada. ADNI was launched in 2003, with clinical assessments and biospecimen collection from $7^{\text {th }}$ September, 2005 to $16^{\text {th }}$ June, 2016. The primary goal of ADNI has been to test whether serial MRI, PET, other biological markers, and clinical and neuropsychological assessments can be

115 combined to measure the progression of mild cognitive impairment (MCI) and early AD. AD and MCI classification followed the criteria described elsewhere $[22,23]$. The ADNI inclusion/exclusion criteria are described in detail at www.adni-info.org. Informed consent was provided by the enrolled participants or their authorized representatives. The ADNI study was approved by the local Institutional Review Boards at the participating centers. In addition, the present study was performed in accordance with the Strengthening the Reporting of Observational Studies in Epidemiology (STROBE) reporting guideline [24].

This study was based on participants with available plasma p-tau181 data (accessed on 20th June, 2020). The time at first plasma p-tau181 measurement defined the baseline time point of our study, which was used for the cross-sectional analyses, as well as the time of diagnostic classification. Longitudinal plasma p-tau181 data (for up to 96 months from baseline) was also evaluated. The number of time points varied between subjects, with the average being 3.11 (median= 3) visits per subject. For a detailed description of longitudinal data, see the Supplementary Appendix. Additional biomarkers assessing cognition, amyloid and tau pathologies as well as neurodegeneration were also investigated cross-sectionally and longitudinally even though they were available for subsets of the cohort, as described below.

\section{Plasma measurements}

Blood samples were collected, processed, stored and analyzed as described previously [25, 26]. Plasma ptau181 was measured using a clinically validated in-house assay described previously [13]. Plasma p-tau181 was measured on Simoa HD-X instruments (Quanterix, Billerica, MA, USA) in April 2020 at the Clinical Neurochemistry Laboratory, University of Gothenburg, Mölndal, Sweden, by scientists blinded to participants'

135 clinical information. Plasma p-tau181 data was collected over 47 analytical runs. Assay precision was assessed by measuring two different quality control samples at the start and end of each run, resulting in within-run and between-run coefficients of variation of 3.3\%-11.6\% and 6.4\%-12.7\%, respectively (Supplementary Table S1). A third quality control sample was used as internal calibrator. Out of 3762 ADNI plasma samples, four were not analyzed due to inadequate volumes. The remaining 3758 all measured above the assay's lower limit of detection $140 \quad(0.25 \mathrm{pg} / \mathrm{mL})$, with only six below the lower limit of quantification $(1.0 \mathrm{pg} / \mathrm{mL})$. 


\section{Other biochemical measurements}

CSF $A \beta_{42}$, p-tau181 and t-tau were measured using the fully automated Elecsys assays (Roche Diagnostics) [27]. In this study, CSF data were matched with plasma biomarker data collected on the same study visit. Plasma neurofilament light (NfL) was measured in the same subjects as p-tau181 at the Clinical Neurochemistry Laboratory, University of Gothenburg, Mölndal, Sweden, using an in-house Simoa immunoassay, as previously described $[25,26]$.

\section{Cognition tests}

Cognitive performance was assessed using the sum of boxes of the Clinical Dementia Rating (CDR-SOB), the Mini-Mental State Examination (MMSE) and the Alzheimer's Disease Assessment Scale-Cognitive Subscale (ADAS-Cog). Cognitive scores were matched with plasma p-tau181 data based on the study visit.

\section{Neuroimaging}

MRI and PET acquisitions followed ADNI protocols (http://adni.loni.usc.edu/methods). The MRI T1weighted images underwent initial preprocessing with intensity normalization and gradient unwarping. They were processed using DARTEL and registered using a six-parameter affine transformation and nonlinearly spatially normalized to the ADNI template. PET images were pre-processed to have an effective point spread function of fullwidth at half-maximum of $8 \mathrm{~mm}$. Subsequently, linear registration and nonlinear normalization to the ADNI template were performed with the transformations deriving from the automatic PET to MRI transformation and the individual's anatomic MRI co-registration. Brain A $\beta$ load was estimated using $\left[{ }^{18} \mathrm{~F}\right]$ florbetapir (A $\beta$ PET), tau load using $\left[{ }^{18} \mathrm{~F}\right]$ flortaucipir (tau PET) and glucose uptake using $\left[{ }^{18} \mathrm{~F}\right]$ fluorodeoxyglucose (FDG PET) standardized uptake value ratios (SUVR). SUVR volumes were generated using the full cerebellum as reference region for $\left[{ }^{18} \mathrm{~F}\right]$ florbetapir, the cerebellar gray for $\left[{ }^{18} \mathrm{~F}\right]$ flortaucipir, and cerebellar vermis and the pons as the reference regions for $\left[{ }^{18} \mathrm{~F}\right]$ fluorodeoxyglucose. Summary PET measures include average SUVR of the meta-ROI regions for tau PET [28], the average SUVR of precuneus, cingulate, inferior parietal, medial prefrontal, lateral temporal, and orbitofrontal cortices for amyloid and the average of the bilateral angular gyrus, bilateral posterior cingulate, and bilateral inferior temporal gyrus for FDG PET [29]. Specific for tau PET, there was a large difference in time between first blood collection and the scan acquisition, and to account for this variability, we used the residuals of tau PET SUVR regressed on the time difference between the two measurements in the analysis.

Brain atrophy was estimated using hippocampal, whole brain and ventricular volumes. These measurements were estimated using FreeSurfer [30] and were adjusted for total intracranial volume (ICV), as previously described [31]. ICV adjustment was performed using data from all cognitively unimpaired (CU) subjects at baseline.

Here, we used the imaging data with the closest acquisition date to the plasma collection. Details on the number of subjects per PET modality at each time point can be found in the Supplementary Appendix.

\section{Cut-points}

The study participants were further classified by their clinical diagnosis and $\mathrm{A} \beta$ status $(\mathrm{A} \beta+/-)$ defined by A $\beta$ PET. The cut-off for A $\beta$ PET ( $>1.11$ SUVR) was determined using receiver operating characteristic (ROC) 
curve analysis contrasting AD versus CU (Youden index). The cut-off definition for CSF p-tau181 ( $>27 \mathrm{ng} / \mathrm{L}$ ) has been previously described [25].

\section{Statistical analysis}

All statistical analyses were performed in R statistical platform v.3.6.3 [32]. Demographic comparisons were done using chi-square test for categorical variables and one-way analysis of variance (ANOVA) followed by Tukey's post-hoc test for continuous variables. Linear regression models (LM) tested the associations between plasma ptau181 concentrations and other variables at baseline, always adjusting for age and gender. Cross-sectional group differences were also evaluated using linear regressions, adjusting again for age and sex, and Tukey's honest significance test was used for post hoc pairwise comparisons, when necessary.

Longitudinal data were also evaluated with linear mixed-effect (LME) models, which always included random intercept and slopes and were adjusted for age, gender and baseline measures when needed. The models were fit using maximum likelihood estimation and time was set as continuous variable. We first compared plasma ptau181 progression between categorical groups. Then, baseline plasma p-tau181 (continuous) was associated with longitudinal data for the other measures. The predictive power of baseline plasma p-tau181 (categorical) was evaluated by examining the difference between p-tau181 positive and negative groups in relation to cognitive decline and progressive neurodegeneration.

Biomarker rate of change was calculated using individual random slopes from LME models including random intercept and slopes and adjusted for age, gender. The rates of change were then correlated using Pearson's correlation coefficient.

Receiver operating characteristic (ROC) curve analysis was used to assess diagnostic accuracies (CU vs AD) and to define the cut-off for plasma p-tau181. ROC 95\% confidence intervals (CI) of sensitivities and specificities were also computed (Youden index).

Cox proportional hazard regression models tested the association between dichotomized values of plasma p-tau181 and the risk of incident AD dementia. The outcome of the model was time to diagnosis and it was adjusted for age and sex. Participants were censored at their last follow-up visit. Hazard ratios (HR) were reported. Schoenfield residuals tested the assumption of proportional hazards and Martingale residuals assessed nonlinearity

The coefficients of variance (CV), and respective confidence intervals, were used to compare within-person p-tau181 variation over time. This analysis was performed using the individual average rate of change, which was calculated by subtracting baseline from follow-up (last visit available) plasma p-tau181 and dividing it by the time difference between the two points.

To facilitate comparison and interpretation of findings, LM and LME were performed using standardized variables when indicated. The Z-scores were based on the mean and standard deviation of the control population. Plasma p-tau181 and NfL were log transformed before standardization.

Were considered outliers subjects in which baseline plasma p-tau181 values were 3 standard deviations (SD) above or below the average of the whole population. These subjects $(\mathrm{n}=8)$ were excluded from the analyses. 


\section{Results}

\section{Demographic characteristics}

In total, 1177 participants were included in the study, 1022 of whom having serial plasma collections resulting in a total of 3758 individual measures of plasma p-tau181. At baseline, 400 participants were clinically classified as $\mathrm{CU}$, 558 as MCI and 219 with $\mathrm{AD}$ dementia. The mean age of the population was 74.1 years $(\mathrm{SD}=7.6)$, with MCI participants being younger on average than $\mathrm{CU}$ and $\mathrm{AD}$ (detailed demographic characteristics are presented in Supplementary Table 2). As expected, AD patients had worse performance in cognitive scores, increased load of A $\beta$ and tau pathologies (evidenced by both CSF and imaging biomarkers), reduced brain metabolism (indexed by FDG PET) and increased brain atrophy as compared to CU and MCI participants. A total of $414(41.4 \%)$ were $A \beta+$. When, stratifying by clinical syndrome, $68(20 \%)$ of the CU, $209(43 \%)$ of the MCI and $137(77 \%)$ of the AD dementia individuals were $\mathrm{A} \beta+$. Overall characteristics of this subsample are further described in Table 1. Given that blood samples were collected and initially stored in multiple sites across North America, we investigated whether this pre-analytical variability would impact plasma p-tau181 quantification. Considering that sites contribute with diverse proportions of participants at each diagnostic group and to avoid the statistical bias this could add in the analysis, we evaluated the effect of the different collection sites (categorical variable) on plasma p-tau181 levels within diagnostic groups. We found that the different sites had, overall, no effect on plasma p-tau181 measurements $\left(f_{\mathrm{CUneg}}=1.28, P_{\mathrm{CUneg}}=0.10 ; f_{\mathrm{CUpos}}=1.07, P_{\mathrm{CUpos}}=0.42 ; f_{\mathrm{MCIneg}}=1.00, P_{\mathrm{MCIneg}}=0.46 ; \quad f_{\mathrm{MCIpos}}=1.23\right.$, $\left.P_{\mathrm{MCIpos}}=0.16 ; f_{\mathrm{AD}}=1.06, P_{\mathrm{AD}}=0.37\right)$.

Longitudinal plasma p-tau181 concentrations were available for 1022 individuals, including $361 \mathrm{CU}$ (16\% $\mathrm{A} \beta+), 508 \mathrm{MCI}(38 \% \mathrm{~A} \beta+)$ and $153 \mathrm{AD}$ dementia (67\% A $\beta+$; Supplementary Table 3). The number of visits per participant ranged from 1 to 5 , being the median $3(\mathrm{CU}=3 ; \mathrm{MCI}=4 ; \mathrm{AD}=2)$. As the majority of the available plasma p-tau181 data ranges between 0-48 months only these values were considered for longitudinal analysis (Supplementary Fig. 1). Further details on the number of participants with longitudinal biomarkers and cognitive measures are described in described in Supplementary Table 3.

\section{Plasma p-tau181 is increased along the Alzheimer's continuum}

In accordance with previous reports, baseline plasma p-tau181 concentrations were significantly higher in $\mathrm{AD}$ dementia (mean=23.6 $\mathrm{pg} / \mathrm{mL}, \mathrm{SD}= \pm 8.8$ ) and $\mathrm{MCI}$ (mean=18.3 $\mathrm{pg} / \mathrm{mL}, \mathrm{SD}= \pm 10.8$ ) as compared to the $\mathrm{CU}$ group (mean=14.9 pg/mL, $\mathrm{SD}= \pm 9.0 ; P<0.0001 ;$ Fig. $1 \mathrm{~A}$ ), irrespective of $\mathrm{A} \beta$ status. The higher plasma $\mathrm{p}$-tau181 concentration in $\mathrm{AD}$ dementia as compared to the $\mathrm{MCI}$ group was highly significant $(P<0.0001)$.

When considering $\mathrm{A} \beta$ status (Fig. 1b), plasma p-tau181 was higher in $\mathrm{A} \beta+\mathrm{CU}, \mathrm{MCI} A \beta+$ and $\mathrm{AD} A \beta+$ than A $\beta$ - CU (all $P<0.0001$ ). Within the same clinical classification, plasma p-tau181 was higher in participants classified as $\mathrm{A} \beta+$ as compared to those determined as $\mathrm{A} \beta$ - (CU, $P<0.0001$; $\mathrm{MCI}, P<0.0001$; $\mathrm{AD}, P=0.003)$. Plasma p-tau181 was also increased in MCI A $\beta+$ and $\mathrm{AD} A \beta+$ compared with $\mathrm{A} \beta+\mathrm{CU}$ (both $P<0.0001$ ).

In addition to age and sex, the models above described were again tested including APOE- $\varepsilon 4$ status as covariate, which did not alter interpretation of the results. 


\section{The diagnostic performance of plasma p-tau181}

To test plasma p-tau181 accuracy in distinguishing clinically and biomarker-defined diagnostic groups, ROC tests compared $A \beta+A D$ dementia patients against all other groups (Fig. 1c). Plasma p-tau181 differentiated $\mathrm{A} \beta+\mathrm{AD}$ from $\mathrm{A} \beta-\mathrm{CU}(\mathrm{AUC}=85.3 \%$; 95\% CI, 81.4\%-89.2\%) and $\mathrm{A} \beta-\mathrm{MCI}(\mathrm{AUC}=83.8 \%$; 95\% CI, 79.8\%-87.7\%). Importantly, plasma p-tau181 distinguished $\mathrm{A} \beta+\mathrm{MCI}$ from $\mathrm{A} \beta$ - $\mathrm{MCI}(\mathrm{AUC}=79.9 \% ; 95 \% \mathrm{CI}, 72.5 \%-82.8 \%)$ and also $\mathrm{A} \beta+\mathrm{CU}$ from $\mathrm{A} \beta$ - $\mathrm{CU}(\mathrm{AUC}=70.4 \% ; 95 \% \mathrm{CI}, 61.9 \%$-78.9\%). In addition, plasma $\mathrm{p}$-tau181 distinguished $\mathrm{A} \beta+$ $A D$ from $A \beta-A D(A U C=70.3 \%$; 95\% CI, 60.8\%-79.8\%). Plasma p-tau181 separated $A \beta+A D$ from all $A \beta-$ participants (AUC=83.5\%; 95\% CI, 80.2\%-86.9\%). Using a cut-off value of $17.7 \mathrm{pg} / \mathrm{mL}$ (generated from ROC analyses, comparing $\mathrm{A} \beta+\mathrm{AD}$ dementia and $\mathrm{A} \beta-\mathrm{CU})$ classified $44.1 \%(\mathrm{n}=521)$ of the participants as positive for plasma p-tau181. Analysis of concordance showed that $28.5 \%(\mathrm{n}=285)$ of the participants were positive concordant with A $\beta$ PET and 27.4\% ( $\mathrm{n}=245$ ) concordant with CSF p-tau181 (Supplementary Fig. 2). Participants who tested negative for plasma p-tau181 were $55.8 \%(\mathrm{n}=659)$ of the study population. Concordant negative cases were $42.8 \%$ $(\mathrm{n}=428)$ and $41.4 \%(\mathrm{n}=367)$ in relation to A $\beta$ PET and CSF p-tau181, respectively. Discordant data were $28.7 \%$ $(\mathrm{n}=286)$ in relation to A PET and $31.2 \%(\mathrm{n}=279)$ in relation to CSF $\mathrm{p}$-tau181. A cut point to define $\mathrm{A} \beta$ positivity based on plasma p-tau181 was also calculated by comparing individuals positive and negative for A $\beta$ PET. Applying a cut-off value of $14.5 \mathrm{pg} / \mathrm{mL}$ (AUC=76.9\%; 95\% CI, 74.0\%-79.8\%) classified $42.1 \%$ of the CU, 56.1\% of $\mathrm{MCI}$ and $85.4 \%$ of the $\mathrm{AD}$ participants as positive for $\mathrm{A} \beta$ pathology.

Next, we evaluated the accuracy of plasma p-tau181 to identify A $\beta+$ at differing stages of the Alzheimer's continuum and compared them to reference standard plasma, CSF and imaging biomarkers, in the same participants. Firstly, and as stated above, plasma p-tau181 had a good performance in separating $A \beta+A D$ dementia and $A \beta+C U$ with comparable AUCs as compared with CSF p-tau181, CSF A $\beta_{42}$ and hippocampal volume (Fig. 1d). Plasma ptau181 outperformed plasma NfL in this comparison (Fig. 1d). We then tested the performance of plasma p-tau181 to identify $\mathrm{A} \beta+$ in $\mathrm{CU}$ and $\mathrm{MCI}$ groups. In this analysis, plasma p-tau181 demonstrated a significantly higher AUC than plasma NfL in identifying $A \beta+$ participants at both stages (Fig. 1e-f), and also in identifying misclassified $A D$ e.g., $\mathrm{A} \beta-\mathrm{AD}$ (p-tau181, $\mathrm{AUC}=70.3$; NfL, $\mathrm{AUC}=54.9$ ). At the $\mathrm{CU}, \mathrm{MCI}$ and dementia stages, plasma $\mathrm{p}$-tau181 also demonstrated higher AUCs in identifying $A \beta$ pathology than hippocampal volume. Plasma $p$-tau181 had marginally lower AUCs in identifying $A \beta+$ cases compared with CSF p-tau181 and CSF $A \beta_{42}$ at both the CU and MCI stages. At the dementia stage, plasma p-tau181 was superior to CSF p-tau181 (AUC=62.6; 95\% CI, 48.9\%-76.4\%) and comparable to $\mathrm{CSF} A \beta_{42}(\mathrm{AUC}=72.1 ; 95 \% \mathrm{CI}, 59.7 \%-84.4 \%)$ in predicting $\mathrm{A} \beta$ pathology.

Plasma p-tau181 associates cross-sectionally with CSF, plasma, imaging and cognitive biomarkers

At the cross-sectional level, plasma p-tau181 correlated with CSF p-tau181 ( $\left.\mathrm{r}=0.36, P<2.2 \times 10^{-16}\right), \mathrm{A} \beta_{42}(=-$ $\left.0.39, P<2.2 \times 10^{-16}\right)$ and t-tau $\left(\mathrm{r}=0.33 ; P<2.2 \times 10^{-16}\right)$, as well as with plasma NfL ( $\mathrm{r}=0.39 ; P<2.2 \times 10^{-16}$; Supplementary Fig. 3a-h). Increased concentrations of plasma $p$-tau181 were also correlated with high $\mathrm{A} \beta\left(\mathrm{r}=0.42 ; P<2.2 \times 10^{-16}\right)$ and tau PET SUVR $\left(\mathrm{r}=0.26 ; P<2.9 \times 10^{-5}\right)$ and reduced FDG PET SUVR $\left(\mathrm{r}=-0.3 ; P<2.2 \times 10^{-16}\right)$. Brain atrophy was also correlated with plasma $\mathrm{p}$-tau181 as indexed by hippocampal volume $\left(\mathrm{r}=-0.34 ; P<2.2 \times 10^{-16}\right)$, ventricular volume $\left(\mathrm{r}=0.24 ; P<2.2 \times 10^{-16}\right)$ and total brain volume $\left(\mathrm{r}=-0.23 ; P<2.2 \times 10^{-16}\right)$. Further, worse performance in cognitive 
assessments was correlated with higher plasma $\mathrm{p}$-tau181 concentrations $\left(\mathrm{r}_{\mathrm{MMSE}}=-0.3 ; \mathrm{r}_{\mathrm{ADAS}-\mathrm{Cog}}=0.34 ; \mathrm{r}_{\mathrm{CDR}-\mathrm{SOB}}=0.31\right.$; all $\left.P<2.2 \times 10^{-16}\right)$.

Linear models were applied to evaluate the association between plasma p-tau181 and the above-mentioned biomarkers, but now adjusting for age, sex and diagnosis (Fig. 2a-h). After adjusting for covariates, higher plasma ptau181 levels were associated with higher CSF p-tau181 $\left(t=8.78, P=2 \times 10^{-16}\right)$ and $\mathrm{t}$-tau concentrations $(t=7.59, P=$ $\left.7.64 \times 10^{-14}\right)$ and lower $\operatorname{CSF~A~} \beta_{42}$ levels $\left(t=-8.27, P=6.11 \times 10^{-16}\right)$ as expected. In addition, high plasma $p$-tau181 was associated with high brain $\mathrm{A} \beta$ and tau load $\left(t_{\mathrm{A} \beta \mathrm{PET}}=10.39, P_{\mathrm{A} \beta \mathrm{PET}}=2 \times 10^{-16} ; t_{\text {tau PET }}=4.07, P_{\text {tau PET }}=6.15 \times 10^{-5}\right)$ whilst inversely associated with FDG PET $\left(t=-4.91, P=1.02 \times 10^{-6}\right)$. Plasma p-tau181 was associated with measures of brain atrophy (hippocampal volume, $t=-4.47, P=8.48 \times 10^{-6}$; ventricular volume, $t=3.18, P=1.10 \times 10^{-3}$ ), except for total brain volume that was no longer associated with plasma $\mathrm{p}$-tau181 after adjusting for covariates $(t=-1.72, P=0.08)$. Worse cognition was also associated with higher levels of plasma $p$-tau181 $\left(t{ }_{\text {MMSE }}=-4.864, P_{\text {MMSE }}=1.34 \times 10^{-6} ; t\right.$ ADAS-Cog $\left.=5.936, P_{\text {ADAS-Cog }}=4.03 \times 10^{-9} ; t_{\mathrm{CDR}-\mathrm{SOB}}=5.604, P_{\mathrm{CDR}-\mathrm{SOB}}=2.71 \times 10^{-8}\right)$. All linear models described here were also applied within diagnostic groups and results are summarized in Supplementary Table S4. Moreover, these models were re-evaluated adding $A P O E-\varepsilon 4$ status to the other covariates, which did not alter interpretation of the results.

Linear models also compared the association between plasma p-tau181 and all other biomarkers between groups defined according to A $\beta$ PET positivity (Supplementary Fig. 4a-h). Overall, plasma p-tau181 was more strongly associated with worsened phenotypic presentations in the $A \beta+$ compared with the $A \beta$ - group (detailed results in Supplementary Table S5).

\section{Baseline plasma p-tau181 predicts progression to dementia, faster cognitive decline and worsening neurodegeneration}

Survival analysis evaluated the risk of progression to AD dementia considering one's baseline plasma ptau181 status (plasma p-tau181 concentrations $>17.7 \mathrm{pg} / \mathrm{mL}$ were considered positive) and clinical diagnosis. The analysis included 729 participants (283 CU and $446 \mathrm{MCI}$ ) with baseline plasma and CSF p-tau181 and up to 84 months of diagnosis data. High plasma p-tau181 was associated with increased risk of AD dementia in MCI (hazard ratio $[\mathrm{HR}]=22.75,95 \% \mathrm{CI}, 9.90-52.31$; Fig. $3 \mathrm{~b})$ and $\mathrm{CU} A \beta+(\mathrm{HR}=3.25,95 \% \mathrm{CI}, 1.12-9.40)$ as compared with $\mathrm{A} \beta-$ $\mathrm{CU}$ (similar pattern of association was found within a shorter time frame of 48 months as shown in Supplementary

Fig 5). This was seen to be similar to the associations found using CSF p-tau181 ( $\mathrm{HR}_{\mathrm{A} \beta+\mathrm{MCl}}=37.1,95 \% \mathrm{CI}, 15.0$ 91.8; $\mathrm{HR}_{\mathrm{A} \beta+\mathrm{CU}}=5.4,95 \% \mathrm{CI}, 1.8-16.3$, Fig. 3a).

When evaluating the predictive power of plasma p-tau181 to detect changes in downstream biomarkers of pathological progression in CU and MCI, LME analysis showed that participants who were plasma p-tau181positive at baseline, and free from dementia, had faster cognitive decline over 100 months in comparison to CU ptau181-negative individuals in two cognitive tests evaluated here (Fig. 3c-d; for detailed description of the results see Supplementary Table S6). Similarly, higher rates of hippocampal atrophy (over 48 months) were observed in MCI plasma p-tau181-positive at baseline $\left(t=-6.07 ; P=2.15 \times 10^{-9}\right)$ and CU plasma $\mathrm{p}$-tau181-positive at baseline $(t=-$ 2.15; $P=0.03)$, but not MCI plasma $\mathrm{p}$-tau181-negative $(t=-1.14 ; P=0.25)$, compared with those who were CU plasma p-tau181-negative (Fig. 3e). In addition, faster decline in FDG PET uptake over 24 months was observed in plasma 
p-tau181-positive MCI participants ( $\left.t=-6.07 ; P=2.15 \times 10^{-9}\right)$ when compared with CU plasma p-tau181-negative (Fig. 3f). Interestingly, when adding AD dementia subjects to the analysis, one can observe that MCI subjects who were positive for plasma p-tau181 reached follow-up biomarker levels consistent with baseline biomarker levels of the AD dementia group (Supplementary Fig. 6).

The interaction between plasma p-tau181 and APOE- 44 statuses was also briefly investigated by LME models which had as outcome measure the biomarkers mentioned above and age, sex and diagnosis were used as covariates. The effect of the interaction was significant only when hippocampal volume $(t=-2.64, P=0.008)$ and ADAS-Cog $(t=2.34, P=0.01)$ were the outcome measures. On FDG PET and MMSE the interaction had no effect $\left(t_{\mathrm{FDG}}=0.86, P_{\mathrm{FDG}}=0.88 ; t_{\mathrm{MMSE}}=-1.73, P_{\mathrm{MMSE}}=0.08\right)$.

\section{Longitudinal characteristics of plasma p-tau181}

Longitudinal plasma p-tau181 was first analyzed by computing individual slopes (up to 48 months), with the LME model accounting for age and sex. No significant difference was observed between average slopes of diagnostic groups $(t=-0.34, P=0.92)$. Similarly, LME compared plasma p-tau181 progression over 24 months between diagnostic groups (also further classified according to A $\beta$ PET status), and no significant difference was

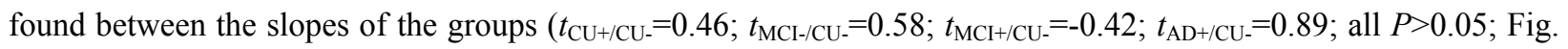
4a). When evaluating the effect of $A P O E$ - $\varepsilon 4$ carriage on plasma p-tau181 progression, the LME adjusted for age, sex and diagnosis showed no difference in the slope of carriers and non-carriers $(t=-0.30 ; P=0.76)$.

To further evaluate the longitudinal relationship between plasma p-tau181 and relevant biomarkers, individual slopes were computed for all variables (adjusting for age and sex), and these values were then correlated with plasma p-tau181 slopes. A positive correlation was found between the slope of CSF p-tau181 and the slope of plasma p-tau181 ( $r=0.1, P=0.04$; Fig $4 \mathrm{~b}$ ). No significant correlation was found between longitudinal changes in plasma p-tau181 and the other biomarkers evaluated (Fig 4c-f). Importantly, when comparing longitudinal changes in CSF p-tau181 with changes in the other biomarkers, similarly to plasma p-tau181, no significant correlations were found, with the exception for A $\beta$ PET (Fig. 4c-f; $\mathrm{r}=0.25, P=1.3 \times 10^{-6}$ ).

To evaluate biomarker stability over time, the average rate of change (Supplementary Fig. 7) was calculated with raw plasma p-tau181 values and this was then used to calculate the coefficient of variance (CV) for each of the diagnostic groups. Plasma p-tau181 was highly stable over the study period, with very low withinperson variability $(\mathrm{CV}: \mathrm{CU}=7 \%, \mathrm{MCI}=9 \%, \mathrm{AD}=12 \%$ ). The $\mathrm{CVs}$ were slightly higher in the respective $\mathrm{A} \beta+$ cases in the $\mathrm{MCI}$ and $\mathrm{AD}$ dementia groups $(\mathrm{A} \beta-\mathrm{CU}(7 \%$, range=6.4\%-7.6\%); $\mathrm{A} \beta+\mathrm{CU}(6 \%$, range $=5.2 \%-7.5 \%) ; \mathrm{A} \beta-\mathrm{MCI}$ $365(7 \%$, range $=6.5 \%-7.5 \%) ; \mathrm{A} \beta+\mathrm{MCI}(12 \%$, range $=10.9 \%-13.3 \%) ; \mathrm{A} \beta-\mathrm{AD}$ dementia $(10 \%$, range $=7.8 \%-13.9 \%)$ and $\mathrm{A} \beta+\mathrm{AD}(14 \%$, range $=12.3 \%-16.3 \%))$. Similar results were found when only considering changes over 24 months, which would reflect the typical time window for therapy in clinical trials: $\mathrm{A} \beta-\mathrm{CU}(9 \%$, range $=8.4 \%-10.1 \%), \mathrm{A} \beta+$ $\mathrm{CU}(7 \%$, range $=5.9 \%-8.6 \%), \mathrm{A} \beta-\mathrm{MCI}(9 \%$, range $=8.2 \%-9.8 \%), \mathrm{A} \beta+\mathrm{MCI}(12 \%$, range $=10.9 \%-13.3 \%), \mathrm{A} \beta-\mathrm{AD}$ $(10 \%$, range $=7.8 \%-13.9 \%)$ and $\mathrm{A} \beta+\mathrm{AD}(14 \%$, range $=12.3 \%-16.3 \%)$.

\section{Discussion}

In this multicenter study, performed in 1177 participants from the ADNI cohort, we verify and confirm the findings from recent single-center cohort studies $[13,17,18]$ that plasma p-tau181 (1) is higher in AD dementia and is 
increased along the Alzheimer's continuum, (2) identifies A $\beta+$ irrespective of disease stage, (3) correlates with CSF p-tau181 and (4) predicts future progression to AD dementia, cognitive decline and hippocampal atrophy. Moreover, utilising the longitudinal aspect of ADNI, we have demonstrated that serial sampling of p-tau181 remains moderately stable overtime, with low individual variability; the small changes detected overtime are significantly related to longitudinal changes in CSF p-tau181 but not any other biomarker modalities.

The significance of the results from this multicentre study, and those from single centre studies that have preceded it, have potentially two major implications. Firstly, the clinical identification of AD dementia could be greatly aided by plasma p-tau181 in primary care, which is important given that discrepancies are common between the clinical diagnosis of AD [33]. In the National Institute of Aging and Alzheimer Association (NIA-AA) Research Framework [34], AD is now defined as a biological construct, documented by biomarker evidence of AD pathology (that is, evidence of both $A \beta$ and tau pathology), and not as a clinical syndrome; thus, plasma p-tau181 could be implemented as a cost-effective and rapid tool to triage possible cases of AD in primary care that would be referred to specialised centers. Moreover, plasma p-tau181 could be used in population-based studies to detect individuals at high risk to develop AD and enrolled them in clinical trials. Plasma p-tau181 has shown to have very high accuracy in determining $\mathrm{AD}$ from non- $\mathrm{AD}$ neurodegenerative diseases (e.g., frontotemporal dementia, progressive supranuclear palsy and cortico-basal syndromes) which is comparable to tau PET [13,17-20]. Although this particular comparison could not be performed in this AD-focused study due to low numbers of subjects with matching plasma p-tau181 and tau PET results, we have shown that individuals who have been clinically defined as $\mathrm{AD}$ dementia or MCI but lack biomarker evidence of $\mathrm{A} \beta$ pathology have significantly lower plasma p-tau181 concentration. However, it is clear that some AD dementia cases without A $\beta$ by PET examination have increased plasma p-tau181 if CSF A $\beta$ has begun to change (Supplementary Fig. 8). This shows great promise that in this clinically challenging scenario, where clinical symptoms are seemingly identical and CSF/PET biomarkers are not available, plasma p-tau181 would provide valuable information to a clinician, which could improve the confidence in an $\mathrm{AD}$ diagnosis and administering symptomatic treatment (e.g., acetylcholinesterase inhibitors or memantine), better inform on patient management, and in the future guide in the selection of patients eligible for diseasemodifying treatments, if such reach the clinic. Moreover, if plasma p-tau181 does not indicate AD, despite cognitive decline, then further investigation could be warranted and implemented at a far earlier stage e.g., FDG PET, dopamine transporter (DAT) or for MRI for frontotemporal dementia, dementia with Lewy bodies or vascular dementia, respectively [35]. This could be accompanied by plasma NfL as increases in this biomarker would indicate on-going neurodegeneration, irrespective of the underlying pathology $[25,26,36]$.

An additional clinical application of plasma p-tau181 is the prediction of progression to dementia in individuals with $\mathrm{MCI}$ or $\mathrm{CU} \mathrm{A} \beta+$. We have demonstrated that plasma $\mathrm{p}$-tau181 accurately predicts the development of dementia, which had a similar predictive performance as CSF p-tau181. This verifies previous findings from the Swedish BioFINDER study [17] but in a multicentric fashion. We also dichotomized participants by MCI and CU to show that individuals with a positive plasma p-tau181 at baseline are still more likely to develop dementia even if no cognitive impairment is present. Importantly, CU individuals with a negative value of plasma p-tau181 at baseline show almost no progression to dementia over a 7 -year period. These findings are further supported by high plasma p-tau181 at baseline being associated with deterioration in cognitive function as assessed with neuropsychiatric 
batteries routinely used in primary care and specialist facilities. We further showed that $\mathrm{A} \beta+$ individuals (MCI or $\mathrm{CU})$ deteriorated at significantly faster rate than the respective $\mathrm{A} \beta$ - individuals. Together, these results support that baseline plasma p-tau181 is highly predictive of future AD diagnosis and AD-mediated cognitive decline.

The second implication would be the use of plasma p-tau181 for participant selection or monitoring in trials that are targeting AD-related brain pathologies or in large-scale epidemiological and genetic studies to identify novel risk and resilience factors for AD. As previously discussed, we and others have shown the ability to demonstrated the presence of $\mathrm{A} \beta$ pathology at the MCI stage with relatively good accuracy ( $\mathrm{AUC}=79.9 \%$ ), which was only slightly inferior to CSF $A \beta_{42}$ and $\mathrm{CSF}$ p-tau181 ( $\mathrm{AUC}=83.2 \%, \mathrm{AUC}=85.2 \%$, respectively) but vastly outperformed plasma NfL and MRI measures of hippocampal volume (AUC $<66 \%$ ). This has fundamental importance in the design of therapeutic trials targeting individuals at the symptomatic phase of the disease, where a positive plasma ptau181 test could confirm AD or reduce screening failure rate even if individuals fulfil the clinical criteria for AD dementia or MCI. The accuracy of plasma p-tau181 to identify A $\beta+$ subjects at the asymptomatic stage was lower, despite highly significant differences from $A \beta$ - participants, and it was evident that CSF $A \beta_{42}$ and CSF p-tau181 were significantly more sensitive to identify brain $A \beta$ pathology at this stage. While this points towards plasma $p$ tau181 having greater utility at the symptomatic stage of the disease than to detect preclinical pathology, other studies show that plasma p-tau181 robustly increase in the preclinical phase in familial AD mutation carriers [37]. Nonetheless, plasma p-tau181 could still act as a pre-screening aid in clinical trials to enrich an asymptomatic population for greater success by a secondary investigation (e.g., A $\beta$ PET). The large multicentric design of this study allowed for speculation on the cost-benefit analysis of plasma p-tau181 as pre-screening for clinical trials targeting the unimpaired population. Of the $336 \mathrm{CU}$ participants included in this study, 68 demonstrated $\mathrm{A} \beta$ positivity, as indexed by $\left[{ }^{18} \mathrm{~F}\right]$ florbetapir, resulting in a positivity rate of $20 \%$ in the asymptomatic sub-cohort $-10 \%$ lower than previously reported prevalence estimates $[38,39]$. When employing a cut-off specifically estimated for A $\beta$ PET positivity (14.5 pg/mL), we classify $143 \mathrm{CU}$ participants as " $p$-tau181-positive for $A \beta$ " of which 60/68 were actually $\mathrm{A} \beta+$ by PET. Considering the prevalence shown in this study, a typical trial design aiming to recruit 1000 $\mathrm{A} \beta+$ asymptomatic individuals would require $\sim 5000$ individuals scanned at the cost of $\$ 15,000,000$ (assuming a cost of $3000 \$ /$ scan). On the contrary, a pre-screening design using p-tau181 in 5000 participants (estimated $\$ 50 /$ participant) would cost $\$ 250,000$ and would yield 2125 plasma p-tau181-positive tests. These 2125 individuals could subsequently be scanned by $\mathrm{A} \beta$ PET at an estimated cost of $\$ 6,645,000$ to confirm positivity for trial enrolment. Comparatively, the pre-screening approach would save in the region of $\$ 8,300,000$, which is over half of the original cost by employing $A \beta$ PET alone. At this preclinical stage, the addition of plasma $A \beta_{42} / A \beta_{40}$ to $p$-tau181 could be an additional tool that improves the accuracy $A \beta+$ prediction and therefore reduce costs further [17]. In addition to being economically advantageous, plasma p-tau181 pre-screening would be time-saving and also logistically simpler to recruit 5000 individuals (or more) willing to undergo blood sampling compared with PET 445 imaging.

A major requirement for widespread use of the plasma p-tau181 assay is the establishment of cut-off values to harmonise readouts across laboratories and in different populations. In our first publication, we estimated a cutoff of $15.9 \mathrm{pg} / \mathrm{ml}$ from a small discovery cohort $(\mathrm{n}=37)$ which performed favorably in the TRIAD $(\mathrm{n}=226)$ and BioFINDER ( $\mathrm{n}=763)$ validation cohorts [13]. This cut-off was also applicable in unicentre cohorts in subsequent 
publications $[19,20,37]$. We report here a cut-off of $17.7 \mathrm{pg} / \mathrm{ml}$ from the large multicentric ADNI cohort, which is comparable to the previous cut-off generated from a small sample set. These findings support robustness of the assay employed in the ADNI cohort and plasma p-tau181 as a biomarker for routine use across clinical settings and laboratories.

We observed longitudinal stability of plasma p-tau181 over several years, which demonstrates that plasma p-tau181 has low biological variability, and measures are methodologically stable and reliable across repeated samplings. This observation could also be of potential benefit in disease-modifying trials seeking a measurable response to a therapeutic target. In fact, when CSF p-tau181 concentrations from individuals with paired CSF and plasma data were analysed, similar observations were made, including a lack of significant longitudinal association with other biomarkers, although a significant but weak correlation existed for CSF p-tau181 and $\left[{ }^{18} \mathrm{~F}\right]$ florbetapir longitudinal change. These findings are supported by a previous report on the ADNI cohort that showed that it would take at least 6.2 years for CSF p-tau181 concentrations to significantly start altering [40]. Changes in plasma would potentially require longer duration since blood is downstream of CSF with respect to central nervous system metabolites. However, we did demonstrate that the longitudinal slopes of plasma and CSF p-tau181 were significantly correlated suggesting, to some degree, that the subtle changes in CSF are reflected in blood. Toledo and colleagues [40] also reported that CSF p-tau181 increased up to $5.1 \mathrm{pg} / \mathrm{mL}$ per year. As the plasma p-tau181 concentration is around 5\% of that in CSF [13], an increase of around $0.26 \mathrm{pg} / \mathrm{mL}$ per year in blood would be seen. The longitudinal trajectories recorded in the present study were similar to these values.

The high association of plasma p-tau181 with measures of amyloid pathology (CSF A $\beta_{42}$ and A $\beta$ PET) and the higher increases of plasma $p$-tau181 in $A \beta+$ than $A \beta$ - groups suggest potential biological relationships between $\mathrm{A} \beta$ pathology and the secretion of brain-specific p-tau181 into blood, in support of the amyloid cascade hypothesis [41]. Whilst such a conclusion cannot be drawn from this clinical observational study, previous data from transgenic animal models support such a mechanism where p-tau metabolism follows but closely associates with $\mathrm{A} \beta$ changes $[42,43]$. Stable isotope-based kinetic studies in humans have also shown that CSF $p$-tau has higher production rates in $A \beta+$ than $A \beta$ - individuals, and that the rate of tau production associates with amyloid pathology [41]. These studies support the theory that $\mathrm{p}$-tau181 production in CSF is an initial neuronal reaction to A $\beta$ changes. The present clinical study appears to have extended these findings to blood. We demonstrated that baseline blood p-tau181 is increased in relation to $A \beta$ levels across the Alzheimer continuum, beginning from the preclinical stage.

This study is not without limitations. The study lacked sufficient tau PET data at the time of blood collection. Although CSF p-tau181 is an highly accurate diagnostic biomarker for AD, tau PET better reflects the degree of NFT pathology [42] and is a superior diagnostic tool for AD [35]. We also used A $\beta$ PET to classify +/groups. There was a relatively common discordance between CSF and PET biomarkers, meaning that individuals classified as A $\beta$ - by PET could be A $\beta+$ by CSF. Previous reports have demonstrated that changes in A $\beta$ CSF precede alterations in A $\beta$ PET [44], and therefore some individuals classified as $A \beta$ - in this study could have underlying and developing $\mathrm{A} \beta$ pathology. This was most apparent in $\mathrm{A} \beta-\mathrm{AD}$ dementia patients where an unusually large mean and variable spread of plasma p-tau181 levels where observed. However, we have further shown that AD dementia patients classified as "A $\beta$ CSF+ / A $\beta$ PET-" have significantly increased levels of plasma $p$-tau181 than "A $\beta$ CSF- / A $\beta$ PET-" (Supplementary Fig. 8). This further demonstrates that plasma p-tau181 has the ability to 
detect subtle changes in $\mathrm{A} \beta$ status and that for a definitive classification of $\mathrm{A} \beta$-, for plasma p-tau181 studies, both CSF and PET biomarkers should be utilized. Furthermore, high rates of dropout in the AD group precluded extensive analyses of the long-term trajectories of plasma p-tau181 in the most advanced stages of the disease ( $>48$ months). Additionally, the potential effect of comorbidities such as vascular dementia contributing on plasma ptau181 could not be examined. However, vascular dementia is unlikely to confound plasma p-tau181 measures since patients with this form of dementia have low concentrations of both CSF and plasma p-tau181 [13].

In summary, plasma p-tau181 is a promising and accurate diagnostic and prognostic biomarker for $\mathrm{AD}$, encouraging for clinical trial use and can be utilised in symptomatic or asymptomatic populations to considerably lower costs to enrich a population prior to A $\beta$ PET confirmation. Furthermore, the longitudinal, within-person plasma p-tau181 measures were shown to be stable over four years demonstrating a potential utility to evaluate and monitor the effects of novel disease-modifying treatments.

\section{Acknowledgments}

Data collection and sharing was funded by ADNI (NIH \#U01 AG024904) and DOD ADNI (\#W81XWH-122-0012). ADNI is funded by the National Institute on Aging, the National Institute of Biomedical Imaging and Bioengineering, and through generous contributions from the following: AbbVie, Alzheimer's Association; Alzheimer's Drug Discovery Foundation; Araclon Biotech; BioClinica, Inc.; Biogen; Bristol-Myers Squibb Company; CereSpir, Inc.; Cogstate; Eisai Inc.; Elan Pharmaceuticals, Inc.; Eli Lilly and Company; EuroImmun; F. Hoffmann-La Roche Ltd and its affiliated company Genentech, Inc.; Fujirebio; GE Healthcare; IXICO Ltd.; Janssen Alzheimer Immunotherapy Research \& Development, LLC.; Johnson \& Johnson Pharmaceutical Research \& Development LLC.; Lumosity; Lundbeck; Merck \& Co., Inc.; Meso Scale Diagnostics, LLC.; NeuroRx Research; Neurotrack Technologies; Novartis Pharmaceuticals Corporation; Pfizer Inc.; Piramal Imaging; Servier; Takeda Pharmaceutical Company; and Transition Therapeutics. The Canadian Institutes of Health Research is providing funds to support ADNI clinical sites in Canada. Private sector contributions are facilitated by the Foundation for the National Institutes of Health (www.fnih.org). The grantee organization is the Northern California Institute for Research and Education, and the study is coordinated by the Alzheimer's Therapeutic Research Institute at the University of Southern California. ADNI data are disseminated by the Laboratory for Neuro Imaging at the University of Southern California.

TKK holds a Brightfocus fellowship (\#A2020812F), and is further supported by the Swedish Alzheimer Foundation (Alzheimerfonden; \#AF-930627), the Swedish Brain Foundation (Hjärnfonden; \#FO2020-0240), the Swedish Dementia Foundation (Demensförbundet), the Agneta Prytz-Folkes \& Gösta Folkes Foundation (\#202000124), the Aina (Ann) Wallströms and Mary-Ann Sjöbloms Foundation, the Anna Lisa and Brother Björnsson's Foundation, Gamla Tjänarinnor, and the Gun and Bertil Stohnes Foundation. NJA is supported by the Swedish Alzheimer Foundation (Alzheimerfonden; \#AF-931009), the Swedish Brain Foundation (Hjärnfonden), the Agneta Prytz-Folkes \& Gösta Folkes Foundation, and the Swedish Dementia Foundation (Demensförbundet). AS was supported by the Emil Aaltonen Foundation and the Paulo Foundation, and currently receives funding from the Orion Research Foundation. MS-C received funding from the European Union's Horizon 2020 Research and Innovation Program under the Marie Sklodowska-Curie action grant agreement No 752310, and currently receives 
funding from Instituto de Salud Carlos III (PI19/00155) and from the Spanish Ministry of Science, Innovation and Universities (Juan de la Cierva Programme grant IJC2018-037478-I). PR-N is supported by the Weston Brain Institute, the Canadian Institutes of Health Research, the Canadian Consortium on Neurodegeneration in Aging and the Fonds de Recherche du Québec - Santé (FRQS; Chercheur Boursier, and 2020-VICO-279314 TRIAD/BIOVIE Cohort), the CIHR-CCNA Canadian Consortium of Neurodegeneration in Aging, and the Canada Foundation for Innovation (project 34874). KB was supported by the Alzheimer Drug Discovery Foundation (ADDF; \#RDAPB201809-2016615), the Swedish Research Council (\#2017-00915), the Swedish Alzheimer Foundation (\#AF742881), Hjärnfonden, Sweden (\#FO2017-0243), and a grant (\#ALFGBG-715986) from the Swedish state under the agreement between the Swedish government and the County Councils, the ALF-agreement. KB is supported by the Swedish Research Council (\#2017-00915), the Alzheimer Drug Discovery Foundation (ADDF), USA (\#RDAPB-201809-2016615), the Swedish Alzheimer Foundation (\#AF-742881), Hjärnfonden, Sweden (\#FO20170243), the Swedish state under the agreement between the Swedish government and the County Councils, the ALFagreement (\#ALFGBG-715986), and European Union Joint Program for Neurodegenerative Disorders (JPND2019466-236). HZ is a Wallenberg Scholar supported by grants from the Swedish Research Council (\#2018-02532), the European Research Council (\#681712), Swedish State Support for Clinical Research (\#ALFGBG-720931), the Alzheimer Drug Discovery Foundation (ADDF), USA (\#201809-2016862), the European Union's Horizon 2020 research and innovation programme under the Marie Skłodowska-Curie grant agreement No 860197 (MIRIADE), and the UK Dementia Research Institute at UCL.

\section{Author contributions}

TKK, ALB, NJA, PSC, MWW, JQT, PR-N, LMS, KB, and HZ conceptualized the research; TKK, NJA, JLR, AS, MS-C, HK, UA, LMS, KB, and HZ performed plasma p-tau181 measurements, data quality control and data compilation; TKK, ALB, NJA, FL, PSC, AMR, MS, TAP, PR-N, KB, and HZ contributed to data analysis; ALB, FL, PSC, TAP and PR-N developed and implemented algorithms for data analysis; TKK, ALB, NJA, KB, and HZ wrote the original manuscript draft. All authors reviewed, edited and approved the final manuscript for submission.

\section{Conflict of interests}

KB has served as a consultant, at advisory boards, or at data monitoring committees for Abcam, Axon, Biogen, JOMDD/Shimadzu, Julius Clinical, Lilly, MagQu, Novartis, Roche Diagnostics, and Siemens Healthineers. H.Z. has served at scientific advisory boards for Wave, Samumed, CogRx, Siemens Healthineers and Roche Diagnostics and has given open lectures for Alzecure, Fujirebio, and Biogen. $\mathrm{HZ}$ and $\mathrm{KB}$ are co-founders of Brain Biomarker Solutions in Gothenburg AB, a GU Ventures-based platform company at the University of Gothenburg. The other authors declare no competing interests.

\section{Data and materials availability}

The files used in preparing this manuscript are publicly available from http://adni.loni.usc.edu/. All data is available in the main text or the supplementary materials. 


\section{References}

1. Blennow K, Leon MJ de, Zetterberg H. Alzheimer's disease. The Lancet. 2006;368:387-403.

2. Jack CR, Knopman DS, Jagust WJ, Petersen RC, Weiner MW, Aisen PS, et al. Tracking pathophysiological processes in Alzheimer's disease: an updated hypothetical model of dynamic biomarkers. Lancet Neurol. 2013;12:207-216.

3. Ashton NJ, Schöll M, Heurling K, Gkanatsiou E, Portelius E, Höglund K, et al. Update on biomarkers for amyloid pathology in Alzheimer's disease. Biomark Med. 2018;12:799-812.

4. Schöll M, Maass A, Mattsson N, Ashton NJ, Blennow K, Zetterberg H, et al. Biomarkers for tau pathology. Mol Cell Neurosci. 2019;97:18-33.

5. Skillbäck T, Farahmand BY, Rosén C, Mattsson N, Nägga K, Kilander L, et al. Cerebrospinal fluid tau and amyloid- $\beta 1-42$ in patients with dementia. Brain. 2015;138:2716-2731.

6. Hansson O, Zetterberg H, Buchhave P, Londos E, Blennow K, Minthon L. Association between CSF biomarkers and incipient Alzheimer's disease in patients with mild cognitive impairment: a follow-up study. Lancet Neurol. 2006;5:228-234.

7. Hanseeuw BJ, Betensky RA, Jacobs HIL, Schultz AP, Sepulcre J, Becker JA, et al. Association of Amyloid and Tau With Cognition in Preclinical Alzheimer Disease: A Longitudinal Study. JAMA Neurol. 2019;76:915-924.

8. Molinuevo JL, Ayton S, Batrla R, Bednar MM, Bittner T, Cummings J, et al. Current state of Alzheimer's fluid biomarkers. Acta Neuropathol (Berl). 2018;136:821-853.

9. Nakamura A, Kaneko N, Villemagne VL, Kato T, Doecke J, Doré V, et al. High performance plasma amyloid- $\beta$ biomarkers for Alzheimer's disease. Nature. 2018;554:249-254.

10. Schindler SE, Bollinger JG, Ovod V, Mawuenyega KG, Li Y, Gordon BA, et al. High-precision plasma $\beta$ amyloid 42/40 predicts current and future brain amyloidosis. Neurology. 2019;93:e1647-e1659.

11. Janelidze S, Stomrud E, Palmqvist S, Zetterberg H, van Westen D, Jeromin A, et al. Plasma $\beta$-amyloid in Alzheimer's disease and vascular disease. Sci Rep. 2016;6:26801.

12. Mattsson N, Zetterberg H, Janelidze S, Insel PS, Andreasson U, Stomrud E, et al. Plasma tau in Alzheimer disease. Neurology. 2016;87:1827-1835.

13. Karikari TK, Pascoal TA, Ashton NJ, Janelidze S, Benedet AL, Rodriguez JL, et al. Blood phosphorylated tau 181 as a biomarker for Alzheimer's disease: a diagnostic performance and prediction modelling study using data from four prospective cohorts. Lancet Neurol. 2020;19:422-433.

14. Tatebe H, Kasai T, Ohmichi T, Kishi Y, Kakeya T, Waragai M, et al. Quantification of plasma phosphorylated tau to use as a biomarker for brain Alzheimer pathology: pilot case-control studies including patients with Alzheimer's disease and down syndrome. Mol Neurodegener. 2017;12.

15. Mielke MM, Hagen CE, Xu J, Chai X, Vemuri P, Lowe VJ, et al. Plasma phospho-tau181 increases with Alzheimer's disease clinical severity and is associated with tau- and amyloid-positron emission tomography. Alzheimers Dement. 2018;14:989-997.

16. Yang C-C, Chiu M-J, Chen T-F, Chang H-L, Liu B-H, Yang S-Y. Assay of Plasma Phosphorylated Tau Protein (Threonine 181) and Total Tau Protein in Early-Stage Alzheimer's Disease. J Alzheimers Dis. 2018;61:1323-1332.

17. Janelidze S, Mattsson N, Palmqvist S, Smith R, Beach TG, Serrano GE, et al. Plasma P-tau181 in Alzheimer's disease: relationship to other biomarkers, differential diagnosis, neuropathology and longitudinal progression to Alzheimer's dementia. Nat Med. 2020;26:379-386.

18. Thijssen EH, La Joie R, Wolf A, Strom A, Wang P, Iaccarino L, et al. Diagnostic value of plasma phosphorylated tau181 in Alzheimer's disease and frontotemporal lobar degeneration. Nat Med. 2020;26:387397.

19. Benussi A, Karikari TK, Ashton NJ, Gazzina S, Premi E, Benussi L, et al. Diagnostic and prognostic value of serum NfL and p-Tau181 in Frontotemporal Lobar Degeneration. J Neurol Neurosurg Psychiatry. 2020;in press.

20. Lantero Rodriguez J, Karikari TK, Suárez-Calvet M, Troakes C, King A, Emersic A, et al. Plasma p-tau181 accurately predicts Alzheimer's disease pathology at least 8 years prior to post-mortem and improves the clinical characterisation of cognitive decline. Acta Neuropathol (Berl). 2020. 27 July 2020. https://doi.org/10.1007/s00401-020-02195-x.

21. Jack CR. The transformative potential of plasma phosphorylated tau. Lancet Neurol. 2020;19:373-374.

22. Petersen RC, Aisen PS, Beckett LA, Donohue MC, Gamst AC, Harvey DJ, et al. Alzheimer's Disease Neuroimaging Initiative (ADNI). Neurology. 2010;74:201-209. 
23. McKhann G, Drachman D, Folstein M, Katzman R, Price D, Stadlan EM. Clinical diagnosis of Alzheimer's disease: report of the NINCDS-ADRDA Work Group under the auspices of Department of Health and Human Services Task Force on Alzheimer's Disease. Neurology. 1984;34:939-944.

24. Elm E von, Altman DG, Egger M, Pocock SJ, Gøtzsche PC, Vandenbroucke JP. The Strengthening the Reporting of Observational Studies in Epidemiology (STROBE) statement: guidelines for reporting observational studies. The Lancet. 2007;370:1453-1457.

25. Mattsson N, Cullen NC, Andreasson U, Zetterberg H, Blennow K. Association Between Longitudinal Plasma Neurofilament Light and Neurodegeneration in Patients With Alzheimer Disease. JAMA Neurol. 2019;76:791-799.

26. Mattsson N, Andreasson U, Zetterberg H, Blennow K. Association of Plasma Neurofilament Light With Neurodegeneration in Patients With Alzheimer Disease. JAMA Neurol. 2017;74:557-566.

27. Hansson O, Seibyl J, Stomrud E, Zetterberg H, Trojanowski JQ, Bittner T, et al. CSF biomarkers of Alzheimer's disease concord with amyloid- $\beta$ PET and predict clinical progression: A study of fully automated immunoassays in BioFINDER and ADNI cohorts. Alzheimers Dement. 2018;14:1470-1481.

28. Jack CR, Wiste HJ, Weigand SD, Therneau TM, Lowe VJ, Knopman DS, et al. Defining imaging biomarker cut points for brain aging and Alzheimer's disease. Alzheimers Dement. 2017;13:205-216.

29. Landau SM, Harvey D, Madison CM, Koeppe RA, Reiman EM, Foster NL, et al. Associations between cognitive, functional, and FDG-PET measures of decline in AD and MCI. Neurobiol Aging. 2011;32:12071218.

30. Fischl B. FreeSurfer. NeuroImage. 2012;62:774-781.

31. Hansen TI, Brezova V, Eikenes L, Håberg A, Vangberg TR. How Does the Accuracy of Intracranial Volume Measurements Affect Normalized Brain Volumes? Sample Size Estimates Based on 966 Subjects from the HUNT MRI Cohort. Am J Neuroradiol. 2015;36:1450-1456.

32. R Core Team. R Core Team (2019). R: A language and environment for statistical computing. R Foundation for Statistical Computing, Vienna, Austria. URL https://www.R-project.org/. 2019. https://www.Rproject.org/.

33. Beach TG, Monsell SE, Phillips LE, Kukull W. Accuracy of the clinical diagnosis of Alzheimer disease at National Institute on Aging Alzheimer Disease Centers, 2005-2010. J Neuropathol Exp Neurol. 2012;71:266273.

34. Jack CR, Bennett DA, Blennow K, Carrillo MC, Dunn B, Haeberlein SB, et al. NIA-AA Research Framework: Toward a biological definition of Alzheimer's disease. Alzheimers Dement. 2018;14:535-562.

35. Leuzy A, Smith R, Ossenkoppele R, Santillo A, Borroni E, Klein G, et al. Diagnostic Performance of RO948 F 18 Tau Positron Emission Tomography in the Differentiation of Alzheimer Disease From Other Neurodegenerative Disorders. JAMA Neurol. 2020. 11 May 2020. https://doi.org/10.1001/jamaneurol.2020.0989.

36. Hansson O, Janelidze S, Hall S, Magdalinou N, Lees AJ, Andreasson U, et al. Blood-based NfL: A biomarker for differential diagnosis of parkinsonian disorder. Neurology. 2017;88:930-937.

37. O'Connor A, Karikari TK, Poole T, Ashton NJ, Lantero Rodriguez J, Khatun A, et al. Plasma phospho-tau181 in presymptomatic and symptomatic familial Alzheimer's disease: a longitudinal cohort study. Mol Psychiatry. 2020:1-10.

38. Jansen WJ, Ossenkoppele R, Knol DL, Tijms BM, Scheltens P, Verhey FRJ, et al. Prevalence of Cerebral Amyloid Pathology in Persons Without Dementia: A Meta-analysis. JAMA. 2015;313:1924-1938.

39. Roberts RO, Aakre JA, Kremers WK, Vassilaki M, Knopman DS, Mielke MM, et al. Prevalence and Outcomes of Amyloid Positivity Among Persons Without Dementia in a Longitudinal, Population-Based Setting. JAMA Neurol. 2018;75:970-979.

40. Toledo JB, Xie SX, Trojanowski JQ, Shaw LM. Longitudinal change in CSF Tau and A $\beta$ biomarkers for up to 48 months in ADNI. Acta Neuropathol. 2013;126:659-670.

41. Sato C, Barthélemy NR, Mawuenyega KG, Patterson BW, Gordon BA, Jockel-Balsarotti J, et al. Tau Kinetics in Neurons and the Human Central Nervous System. Neuron. 2018;97:1284-1298.e7.

42. Mattsson-Carlgren N, Andersson E, Janelidze S, Ossenkoppele R, Insel P, Strandberg O, et al. A $\beta$ deposition is associated with increases in soluble and phosphorylated tau that precede a positive Tau PET in Alzheimer's disease. Sci Adv. 2020;6:eaaz2387.

43. Maia LF, Kaeser SA, Reichwald J, Hruscha M, Martus P, Staufenbiel M, et al. Changes in Amyloid- $\beta$ and Tau in the Cerebrospinal Fluid of Transgenic Mice Overexpressing Amyloid Precursor Protein. Sci Transl Med. 2013;5:194re2-194re2. 
44. Palmqvist S, Schöll M, Strandberg O, Mattsson N, Stomrud E, Zetterberg H, et al. Earliest accumulation of $\beta$ amyloid occurs within the default-mode network and concurrently affects brain connectivity. Nat Commun. $2017 ; 8: 1214$.

\section{Figure legends}

685 Fig. 1. Plasma p-tau181 profile. Distribution of plasma p-tau181 concentrations across clinically defined diagnostics groups (a) showing higher biomarker levels associated with symptomatic disease stages. When considering A $\beta$ PET status in addition to clinical diagnosis (b), plasma $p$-tau181 was found at higher concentrations in $A \beta+$ groups. The accuracy of plasma $p$-tau181 in distinguishing $A \beta+A D$ from other diagnostic groups is evidenced by AUCs as shown in (c). In addition, the accuracy of plasma p-tau181 in identifying A $\beta$ pathology was 690 evaluated in the context of other biomarkers (d-f).

Fig. 2. Cross-sectional associations at baseline. Linear regression analyses show that plasma p-taul81 is significantly associated with CSF, plasma and imaging biomarkers within diagnostic groups (a-h). Detailed description of findings are reported in Supplementary Table S4.

695

Figure 3. Plasma p-tau181 as a predictor.

Cox-proportional hazard model showing that higher levels of baseline CSF (a) and plasma (b) p-tau181 are associated with an increased risk to progress to $\mathrm{AD}$ dementia, as evidenced by the Kaplan-Meier curves. Moreover, linear mixed effect models indicated that high baseline plasma p-tau181 levels are associated with worse cognitive performance (c-d) and faster neurodegenerative processes (e-f). The * indicates the longitudinal trajectories that are significantly different from plasma p-tau181 negative CU (the reference group).

Fig. 4. Longitudinal plasma p-tau181 profile. Linear mixed effect models indicate that plasma p-tau181 trajectories over time are similar across diagnostic groups (a). Pearson correlation analysis shows that individual plasma p-tau181 changes over time and are correlated with CSF p-tau181 changes (b). In addition, correlation plots (c-f) suggest comparable associations between CSF and plasma p-tau181 and the other CSF and imaging biomarkers. 
Table 1. Baseline and longitudinal participant characteristics according to clinical diagnosis and Aß PET status.

\begin{tabular}{|c|c|c|c|c|c|c|}
\hline Characteristic a,b & $\begin{array}{l}A \beta-C U \\
(n=268)\end{array}$ & $\begin{array}{l}A \beta+C U \\
(n=68)\end{array}$ & $\begin{array}{l}\mathbf{A \beta}-\mathbf{M C I} \\
(\mathrm{n}=\mathbf{2 7 7})\end{array}$ & $\begin{array}{l}A \beta+M C I \\
(n=209)\end{array}$ & $\begin{array}{l}\text { A } \beta-A D \\
\text { dementia } \\
(n=41)\end{array}$ & $\begin{array}{l}\mathbf{A \beta}+\mathbf{A D} \\
(\mathrm{n}=137)\end{array}$ \\
\hline $\begin{array}{l}\text { Age at baseline, } \\
\text { years }\end{array}$ & $73.5(6.5)$ & $76.9(6.2)$ & $71.4(8.0)^{*, \#}$ & $73.9(6.7)$ & $77.3(7.0)^{*, \#}$ & $73.4(8.2)$ \\
\hline $\begin{array}{l}\text { Sex (female), No. } \\
(\%)\end{array}$ & $131(48.9 \%)$ & $43(63.2 \%)$ & $125(45.1 \%)$ & $87(41.6 \%)$ & $9(22.0 \%)$ & $66(47.8 \%)$ \\
\hline $\begin{array}{l}\geq 1 \text { APOE } \varepsilon 4, \text { No. } \\
(\%)\end{array}$ & $61(22.8 \%)^{*}$ & $33(48.5 \%)^{*, \#}$ & $90(32.3 \%)^{*}$ & $142(67.9 \%)^{\#}$ & $14(34.1 \%)^{*}$ & $108(78.3 \%)^{\#}$ \\
\hline $\begin{array}{l}\text { Educational level, } \\
\text { years }\end{array}$ & $16.7(2.7)^{*}$ & $16.6(2.2)$ & $16.3(2.6)$ & $15.9(2.8)^{\#}$ & $15.8(2.4)$ & $15.7(2.7)^{\#}$ \\
\hline \multicolumn{7}{|c|}{ CSF biomarkers at baseline, mean(SD) } \\
\hline $\mathrm{A} \beta_{42}, \mathrm{pg} / \mathrm{mL}$ & $\begin{array}{l}1143.4(332 \\
.5)^{*}\end{array}$ & $\begin{array}{l}744.4(253.8) \\
*, \#\end{array}$ & $1078.9(353.9)$ & $\begin{array}{l}681.9(196.6) \\
*, \#\end{array}$ & $\begin{array}{l}867.8(392.9) \\
*, \#\end{array}$ & 590.7(187.1) \\
\hline P-tau181, $\mathrm{pg} / \mathrm{mL}$ & $18.0(7.4)^{*}$ & $31.1(12.4)^{*}, \#$ & $18.9(8.7)^{*}$ & \begin{tabular}{|l|}
34.1 \\
$(13.8)^{*, \#}$
\end{tabular} & $33.9(19.8)^{\#}$ & $39.9(15.5)^{\#}$ \\
\hline Total-tau, $\mathrm{pg} / \mathrm{mL}$ & $\begin{array}{l}200.8 \\
(73.8)^{*}\end{array}$ & $\begin{array}{l}310.1(115.2) \\
*, \#\end{array}$ & $207.5(86.6)^{*}$ & \begin{tabular}{|l|}
339.0 \\
$(125.8)^{*}, \#$
\end{tabular} & $\begin{array}{l}348.0 \\
(180.7)^{\#}\end{array}$ & $\begin{array}{l}399.2 \\
(149.6)^{\#}\end{array}$ \\
\hline \multicolumn{7}{|c|}{ Imaging measure at baseline, mean(SD) } \\
\hline $\mathrm{A} \beta$ PET & $0.9(0.1)^{*}$ & $1.3(0.1)^{*, \#}$ & $1.0(0.1)^{*}$ & $1.3(0.1)^{\#}$ & $1.0(0.1)^{*}$ & $1.3(0.1)^{\#}$ \\
\hline $\begin{array}{l}\text { Hippocampal } \\
\text { volume, } \mathrm{mm}^{3}\end{array}$ & $\begin{array}{l}7609.3 \\
(746.6)^{*}\end{array}$ & $\begin{array}{l}7117.6 \\
(763.1)^{*, \#}\end{array}$ & $\begin{array}{l}7114.1 \\
(1077.6)^{*, \#}\end{array}$ & $\begin{array}{l}6655.9 \\
(1025.3)^{*, \#}\end{array}$ & $\begin{array}{l}5709.0 \\
(855.3)^{\#}\end{array}$ & $\begin{array}{l}5914.5 \\
(887.6)^{\#}\end{array}$ \\
\hline Whole brain, $\mathrm{mm}^{3}$ & $\begin{array}{l}1053916.5 \\
(61303.2)^{*}\end{array}$ & $\begin{array}{l}1028401.0 \\
(64418.9)^{*} \\
\end{array}$ & $\begin{array}{l}1055008.6 \\
(66773.0)^{*} \\
\end{array}$ & \begin{tabular}{|l|}
1039169.6 \\
$(62083.3)^{*}$ \\
\end{tabular} & $\begin{array}{l}988329.7 \\
(63365.8)^{\#}\end{array}$ & $\begin{array}{l}998562.3 \\
(61268.6)^{\#}\end{array}$ \\
\hline $\begin{array}{l}\text { Ventricular } \\
\text { volume, } \mathrm{mm}^{3}\end{array}$ & $\begin{array}{l}36000.8(17 \\
561.8)^{*}\end{array}$ & $\begin{array}{l}39765.7 \\
(17164.8)^{*}\end{array}$ & $\begin{array}{l}39421.3 \\
(20891.3)^{*}\end{array}$ & $\begin{array}{l}39369.8 \\
(18233.9)^{*}\end{array}$ & $\begin{array}{l}57963.6 \\
(25203.5)^{\#}\end{array}$ & $\begin{array}{l}47973.4 \\
(18256.9)^{\#}\end{array}$ \\
\hline FDG-PET & $1.3(0.1)^{*}$ & $1.3(0.1)^{*}$ & $1.3(0.1)^{*}$ & $1.2(0.1)^{*}, \#$ & $1.1(0.2)^{*, \#}$ & $1.0(0.1)^{\#}$ \\
\hline \multicolumn{7}{|c|}{ Cognitive score at baseline } \\
\hline MMSE & $29.1(1.3)^{*}$ & $28.8(1.0)^{*}$ & $28.4(1.7)^{*, \#}$ & $27.6(1.9)^{*, \#}$ & $23.7(1.9)^{\#}$ & $22.9(2.7)^{\#}$ \\
\hline CDR-SOB & $0.04(0.1)^{*}$ & $0.2(0.4)^{*}$ & $1.4(0.9)^{*}, \#$ & $1.7(1.1)^{*, \#}$ & $4.2(1.7)^{*, \#}$ & $4.8(2.0)^{\#}$ \\
\hline ADAS-Cog & $5.3(3.0)^{*}$ & $6.2(2.6)^{*}$ & $8.0(3.8)^{*}, \#$ & $10.6(4.5)^{*, \#}$ & $19.8(7.0)^{*, \#}$ & $21.2(7.9)^{\#}$ \\
\hline \multicolumn{7}{|c|}{ Plasma biomarkers at baseline, mean(SD) } \\
\hline $\begin{array}{l}\text { Plasma p-tau181, } \\
\mathrm{pg} / \mathrm{mL}\end{array}$ & $14.2(9.0)^{*}$ & $19.1(8.2)^{*, \#}$ & $14.6(9.9)^{*}$ & $22.8(9.9)^{\#}$ & $19.4(6.5)^{*}$ & $25.5(8.6)^{\#}$ \\
\hline $\begin{array}{l}\text { Plasma NfL, } \\
\mathrm{pg} / \mathrm{mL}\end{array}$ & $35.9(16.6)^{*}$ & $42.4(16.1)$ & $38.2(23.9)^{*}$ & $44.6(23.5)^{\#}$ & $50.9(21.0)^{\#}$ & $46.9(19.6)^{\#}$ \\
\hline
\end{tabular}

${ }^{\text {a }}$ Continuous variables are given as mean (SD)

${ }^{\mathrm{b}} \mathrm{A} \beta$ status defined by $\mathrm{A} \beta$ PET.

${ }^{*} \mathrm{p}<0.05$ compared with the $\mathrm{A} \beta+\mathrm{AD}$ group \# $\mathrm{p}<0.05$ compared with the $\mathrm{A} \beta$ - $\mathrm{CU}$ group

Continuous variables were compared with one-way ANOVA followed by Tukey's post-hoc test. Categorical variables were compared with Chi test 

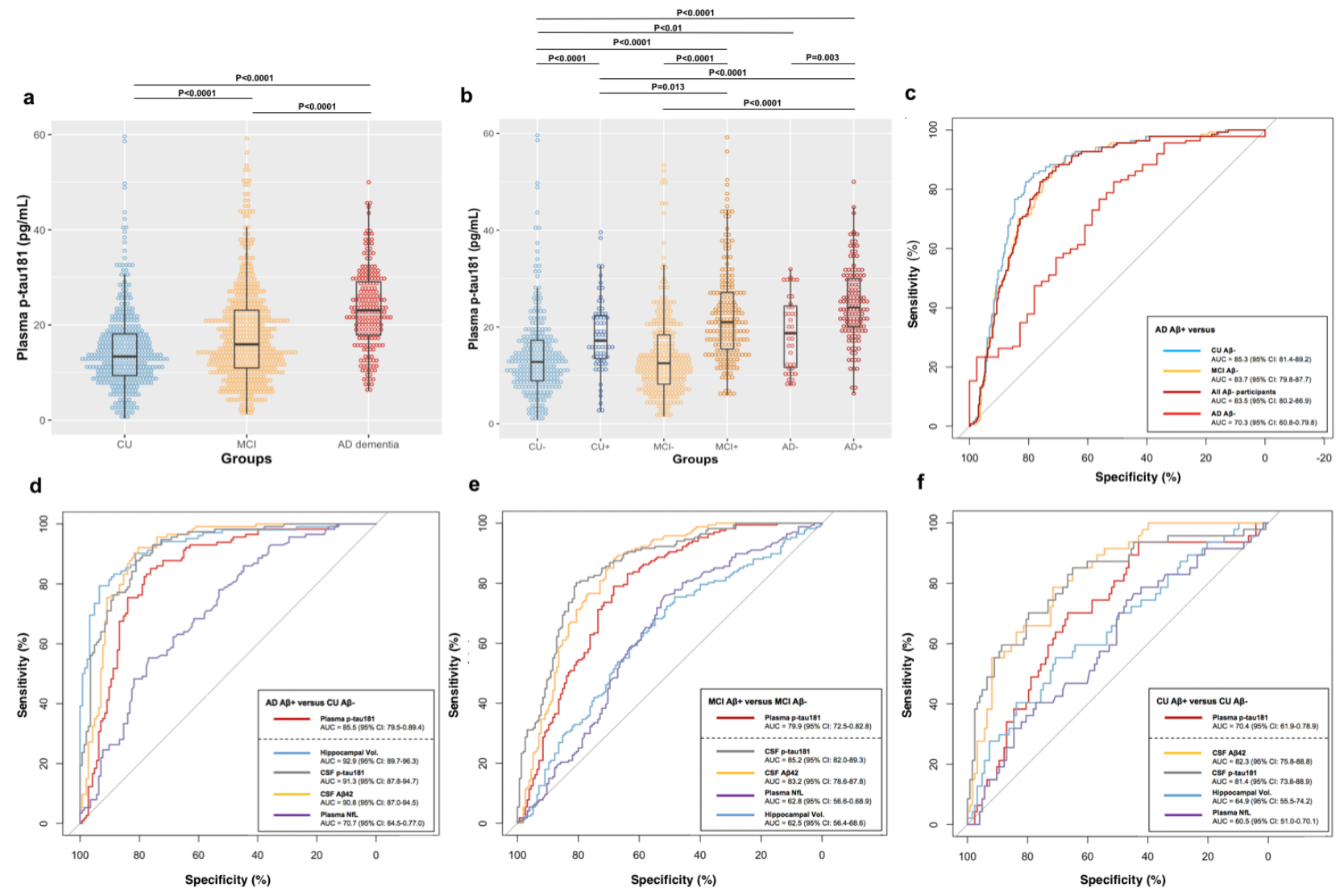

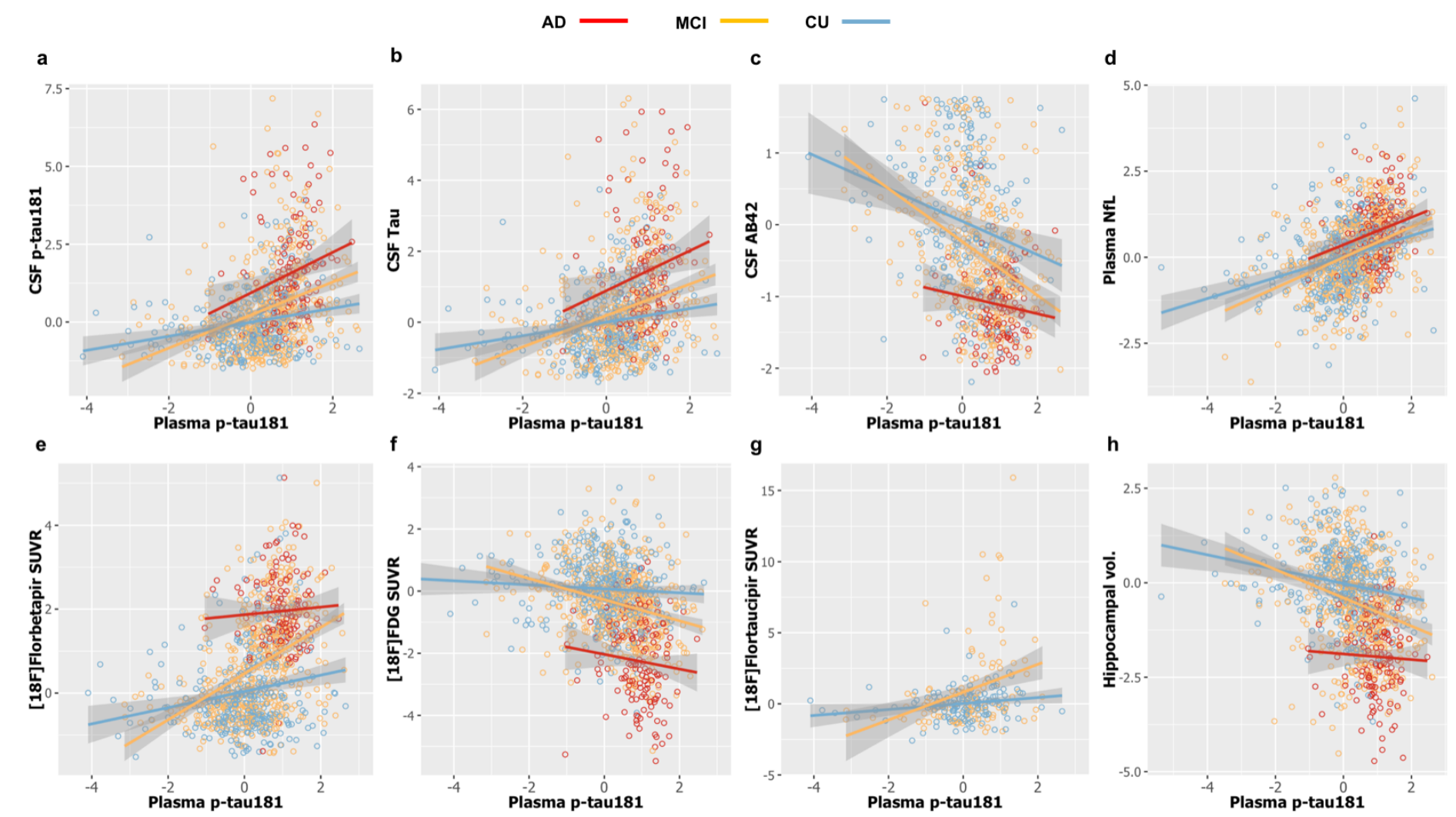
a

Kaplan-Meir Curve - CSF p-tau181

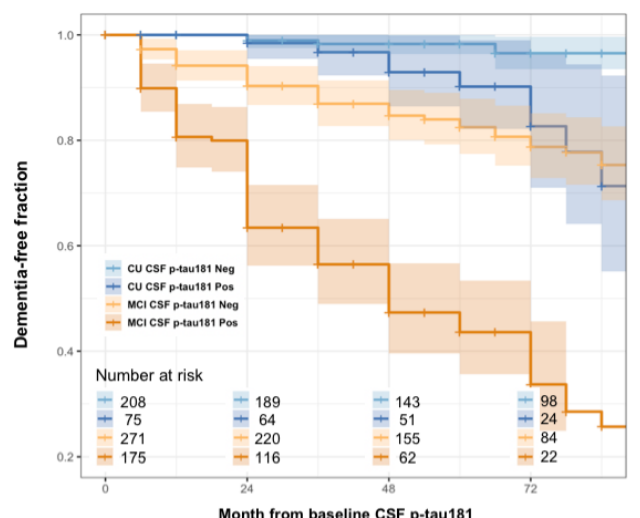

d

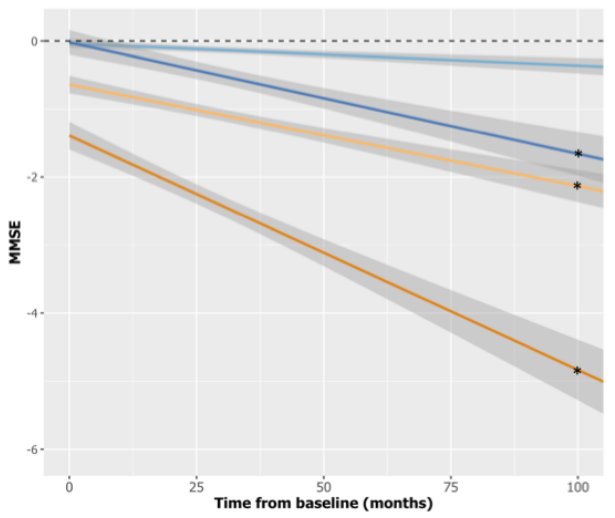

b Kaplan-Meir Curve - Plasma p-tau181

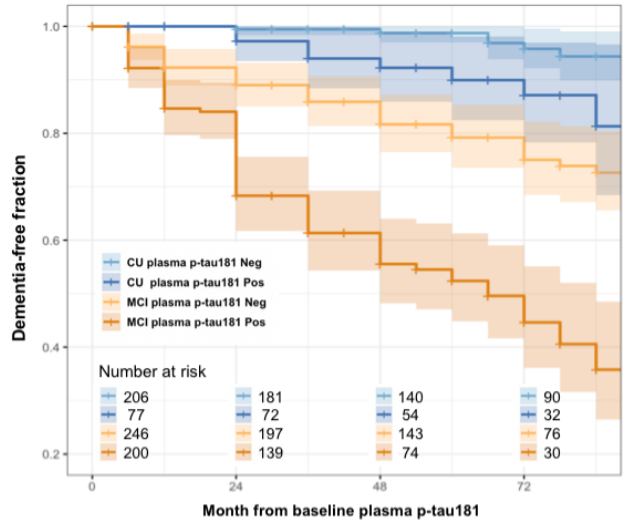

e

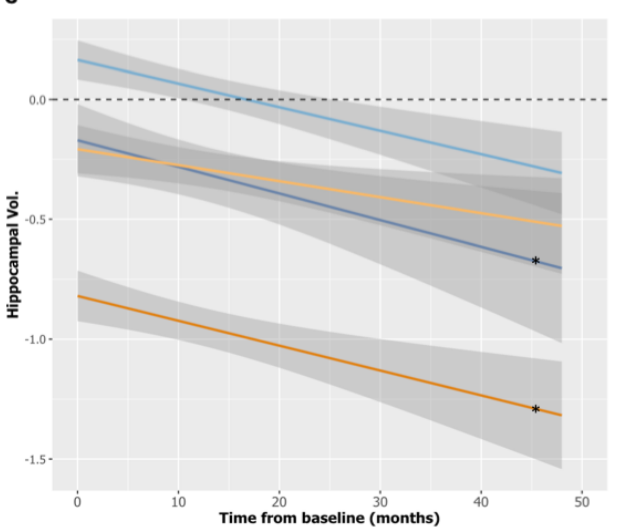

c

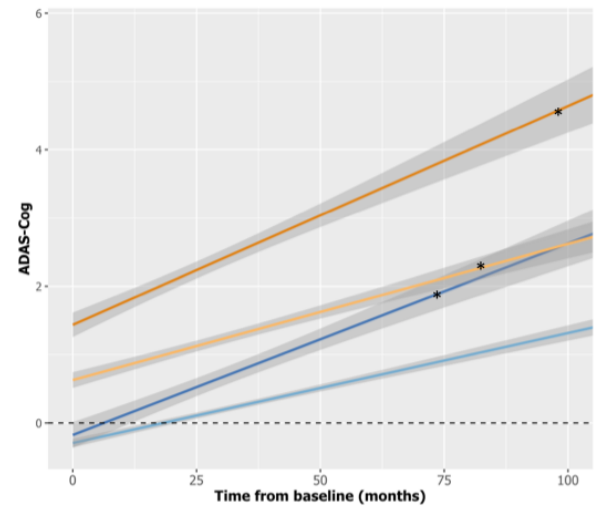

f

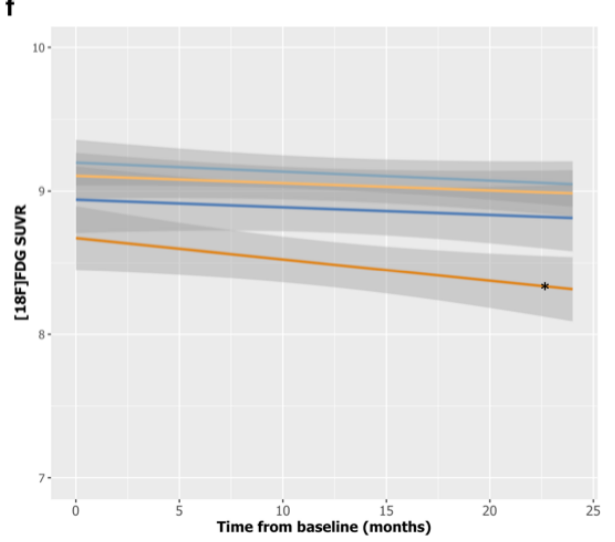



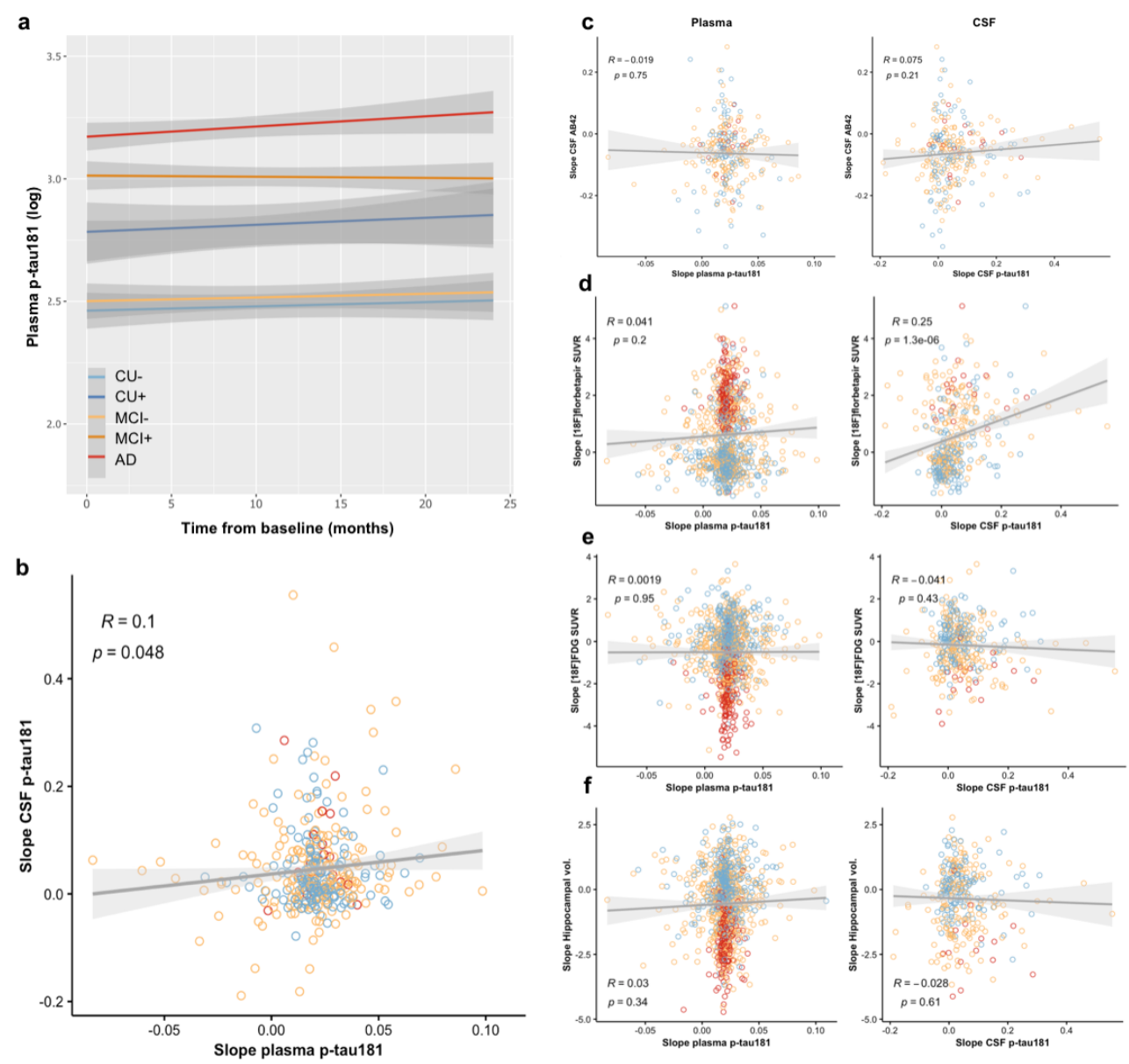